\title{
Numerous aggregates of "tiny" micromegakaryocytes in the bone marrow
}

\author{
Yataro Yoshida Yoko Hamakawa \\ Shigeru Oguma $\cdot$ Tatsuya Katsurada \\ Chisaki Mizumoto $\cdot$ Hitoshi Ohno
}

Received: 17 June 2010/Revised: 22 July 2010/Accepted: 23 July 2010/Published online: 14 August 2010

(C) The Japanese Society of Hematology 2010

A 70-year old male presented with leukocytosis $\left(24.9 \times 10^{9} / \mathrm{L}\right)$ and thrombocytosis $\left(727 \times 10^{9} / \mathrm{L}\right)$. Hb was $14.9 \mathrm{~g} / \mathrm{dL}$, neutrophils $81 \%$, basophils $1 \%$, eosinophils $4 \%$, and myelocytes $1 \%$. He had no splenomegaly. Serum LDH was $477 \mathrm{U} / \mathrm{L}, \mathrm{B}_{12} 755 \mathrm{pg} / \mathrm{m}$, and ferritin $104 \mathrm{ng} / \mathrm{dL}$. Neutrophil alkaline phosphatase activity was normal. The marrow was moderately cellular with many aggregates of micromegakaryocytes, each aggregate comprising up to 20 "tiny" micromegakaryocytes (Fig. 1). Small cells were close to the size of lymphocytes. Although usual-sized micromegakaryocytes were occasionally seen, most "tiny" micromegakaryocytes were found to form aggregates accompanying many platelets (Fig. 2a, b) and such aggregates were scattered in the entire length of marrow film. Immature granulocytes showed hypogranulation (Fig. 3a), and occasional mature cells showed hypersegmentation (Fig. 3b, c) and cytoplasmic Döhle bodies (arrowheads Fig. 3d). Erythroid series showed mild megaloblastoid changes. These features resembled myelodysplastic syndromes (MDS). Blast cells were few. Normal marrow cytogenetic analysis and the lack of BCR-ABL in mRNA analysis excluded chronic myeloid leukemia

Y. Yoshida $(\bowtie) \cdot$ Y. Hamakawa · S. Oguma · T. Katsurada The Center for Hematological Diseases,

Takeda General Hospital, 28-1 Ishida-Moriminami,

Fushimi-Ku, Kyoto 601-1495, Japan

e-mail: yoshida@takedahp.or.jp

C. Mizumoto

Department of Hematology,

Kyoto University, Kyoto, Japan

H. Ohno

Department of Hematology,

Hyogo Prefectural Amagasaki Hospital,

Hyogo, Japan

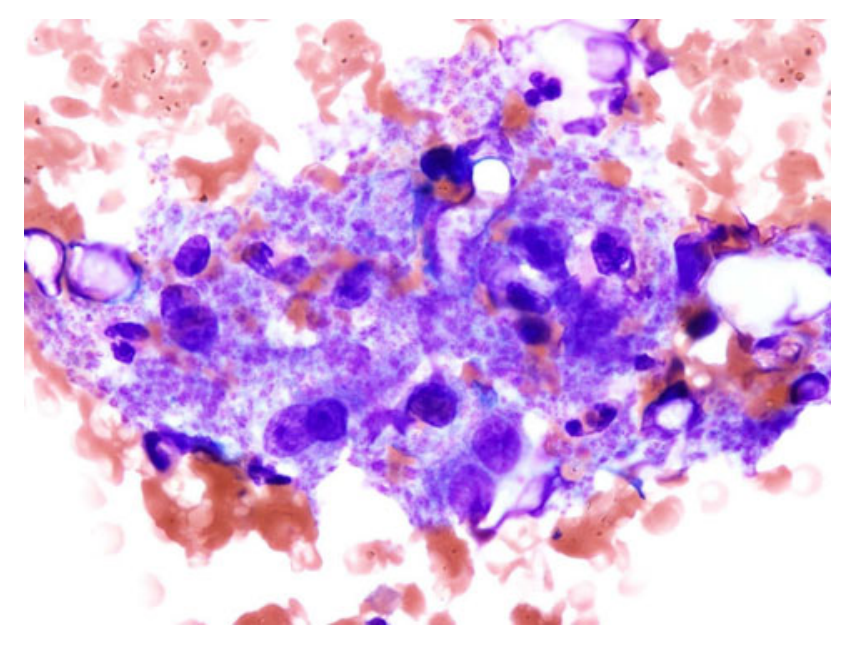

Fig. 1 Moderately cellular marrow with many aggregates of micromegakaryocytes

(CML). Marrow WT1 mRNA level was 1600 copies/ $\mu \mathrm{g}$ RNA. No marrow fibrosis was seen in biopsy. These findings excluded myeloproliferative neoplasm (MPN). Proliferation and marked dysplasia in megakaryocytic and myeloid lineages falls within the scope of MDS/MPN, unclassifiable, according to the current WHO system, rather than atypical BCR-ABL negative CML. The marrow findings remained essentially unchanged in repeat study although blood counts oscillated in response to the intermittent hydroxyurea $(1000 \mathrm{mg})$ treatment. 
Fig. 2 "Tiny"

micromegakaryocytes form aggregates accompanying many platelets
Fig. 3 a Hypogranulation in mature granulocytes, $\mathbf{b}, \mathbf{c}$ hypersegmentation in occasional mature cells, and d cytoplasmic Döhle bodies (arrowheads)
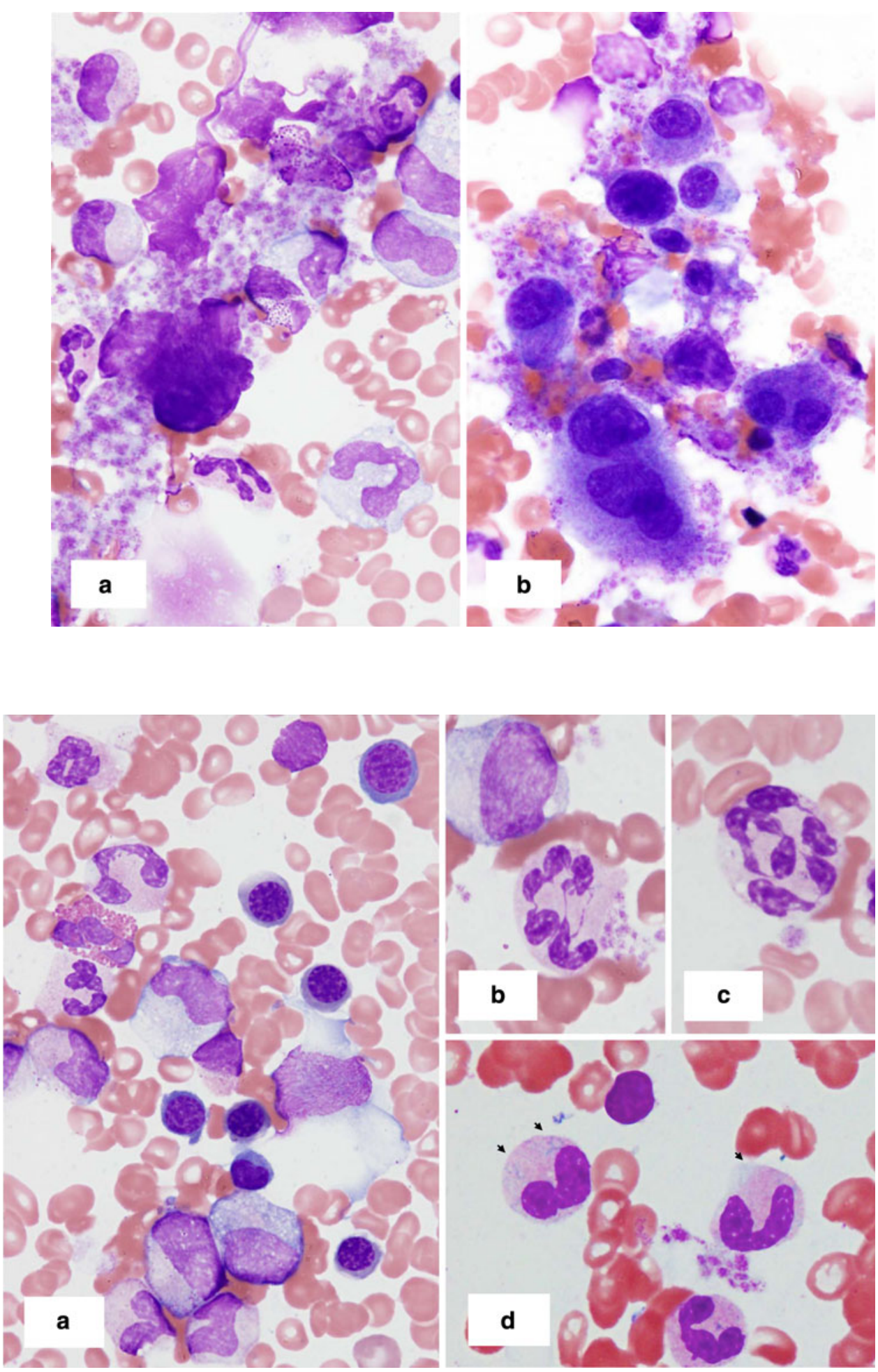